Karla Nazaré Ferreira Damasceno

\title{
Tratamento de Exceções Sensível ao Contexto
}

\section{Dissertação de Mestrado}

Dissertação apresentada como requisito parcial para obtenção do título de Mestre pelo Programa de PósGraduação em Informática da PUC-Rio.

Orientadores: Carlos José Pereira de Lucena Alessandro Fabricio Garcia 
Karla Nazaré Ferreira Damasceno

\section{Tratamento de Exceções Sensível ao Contexto}

Dissertação apresentada como requisito parcial para obtenção do título de Mestre pelo Programa de PósGraduação em Informática da PUC-Rio. Aprovada pela Comissão Examinadora abaixo assinada.

Prof. Carlos José Pereira de Lucena Orientador

Departamento de Informática - PUC-Rio

Prof. Alessandro Fabricio Garcia

Co-orientador Computing Department - Lancaster University - UK

Prof. Arndt von Staa

Departamento de Informática - PUC-Rio

Prof. Firmo Freire

Departamento de Informática - PUC-Rio

Prof. José Eugenio Leal Coordenador Setorial do Centro

Técnico Científico - PUC-Rio

Rio de Janeiro, 31 de março de 2006 
Todos os direitos reservados. É proibida a reprodução total ou parcial do trabalho sem autorização da universidade, da autora e dos orientadores.

\section{Karla Nazaré Ferreira Damasceno}

Graduou-se no Curso de Bacharelado em Ciência da Computação da Universidade Federal do Pará (UFPA) em 2003. É pesquisadora nas áreas de Engenharia de Software de Sistemas Multi-Agentes e Desenvolvimento de Software Orientado a Aspectos no Laboratório de Engenharia de Software (LES) da PUC-Rio.

Ficha Catalográfica

Damasceno, Karla Nazaré Ferreira

Tratamento de exceções sensível ao contexto / Karla Nazaré Ferreira Damasceno ; orientador: Carlos José Pereira de Lucena. - Rio de Janeiro : PUC, Departamento de Informática, 2006.

107 f. : il. ; $30 \mathrm{~cm}$

Dissertação (mestrado) - Pontifícia Universidade Católica do Rio de Janeiro, Departamento de Informática.

Inclui referências bibliográficas.

1. Informática - Teses. 2. Tratamento de exceções. 3. Computação móvel. 4. Sensibilidade ao contexto. 5. Middleware. 6. Tolerância a falhas. I. Lucena, Carlos José Pereira de. II. Pontifícia Universidade Católica do Rio de Janeiro. Departamento de Informática. III. Título. 


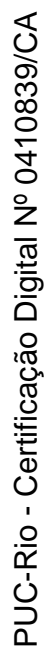

A Deus, cujo conhecimento e amizade dão sentido e orientação a tudo. 


\section{Agradecimentos}

A Deus, pela vida, pela gratuidade de seu amor através de Jesus Cristo e por se utilizar de tudo para me tornar uma pessoa mais madura, feliz e capaz de amar.

À Comunidade Católica Shalom, por sido caminho para intimidade com Deus, vivência fraterna e serviço, me ajudando a não ficar restrita a minha própria vida.

À minha família, especialmente aos meus pais, Ray e Carlos, por todo amor e incentivo. Neste tempo compreendi que em qualquer situação, mesmo a distância, posso contar com vocês sempre.

Às amigas que são minha família no Rio de Janeiro, Cidiane, Mariana e Shirley. À Cidiane, pela generosidade ao partilhar sua vida; sinal da fidelidade de Deus, que realiza maravilhas entre nós. À Mariana, por sua alegria e espontaneidade, que me convidam à coragem de ir ao encontro do outro. À Shirley, por sua presença ter inaugurado um novo tempo em nossas vidas.

A meus amigos, por celebrarem os momentos bons e me incentivarem a ir além do que parecia ser capaz. À Morgana, por valorizar cada detalhe em minha vida. À Cleide, por me impulsionar a prosseguir. À Sônia, pela facilidade para se envolver e amar. À Dacler, por poucos, mas preciosos momentos de partilha. A todos do Laboratório de Engenharia de Software, por enriquecerem o ambiente de trabalho com suas vidas, em especial, Lyrene, Eduardo, Carolina, Míriam e Roberta.

Ao meu orientador Prof $^{\circ}$ Lucena e ao meu co-orientador Alessandro, pelo grande apoio e por me ensinarem a ter um novo olhar a respeito das dificuldades. Sou profundamente grata por ter trabalhado com excelentes profissionais como vocês.

Aos pesquisadores do LAC da PUC-Rio, por sua colaboração e disponibilidade constantes, fundamentais para desenvolver todo este trabalho.

À PUC-Rio, à Fundação Padre Leonel Franca e ao CNPq, pelo auxílio que possibilitou a realização deste trabalho. 


\section{Resumo}

Damasceno, Karla. Tratamento de Exceções Sensível ao Contexto. Rio de Janeiro, 2006. 107p. Dissertação de Mestrado - Departamento de Informática, Pontifícia Universidade Católica do Rio de Janeiro.

Tratamento de erros em aplicações móveis sensíveis ao contexto não é uma tarefa trivial devido às características peculiares destes sistemas, como mobilidade, comunicação assíncrona e aumento de imprevisibilidade. Mecanismos convencionais de tratamento de exceções não podem ser utilizados por vários motivos. Primeiro, a propagação de erros deve considerar as mudanças contextuais que ocorrem constantemente nestes sistemas. Segundo, as atividades de recuperação de erros e a estratégia de tratamento de exceções também precisam freqüentemente ser selecionadas de acordo com as informações de contexto. Terceiro, a própria caracterização de uma exceção pode depender do contexto dos dispositivos envolvidos. Embora vários middlewares orientados a contexto ofereçam suporte ao desenvolvimento de aplicações móveis, estes sistemas raramente fornecem suporte adequado ao tratamento de exceções. Este trabalho realiza uma análise das soluções existentes para tratamento de exceções, considerando os requisitos de sensibilidade ao contexto. Além disso, são propostos um modelo para tratamento de exceções sensível ao contexto e um mecanismo implementado a partir de MoCA (Mobile Collaboration Architecture). MoCA é um middleware publish-subscribe que oferece suporte ao desenvolvimento de aplicações móveis colaborativas através da incorporação de serviços de contexto. Finalmente, este trabalho avalia o mecanismo de exceções proposto através de sua utilização em alguns protótipos de aplicações colaborativas desenvolvidas a partir de MoCA. Através do mecanismo, foram implementadas diferentes estratégias de tratamento de exceções que consideram as informações de contexto das aplicações.

\section{Palavras-chave}

Tratamento de exceções, computação móvel, sensibilidade ao contexto, middleware, tolerância a falhas. 


\section{Abstract}

Damasceno, Karla. Context-Sensitive Exception Handling. Rio de Janeiro, 2006. 107p. Dissertação de Mestrado - Departamento de Informática, Pontifícia Universidade Católica do Rio de Janeiro.

Context-sensitive exception handling on mobile systems is not a trivial task due to their intrinsic characteristics: mobility, asynchrony and increased unpredictability. Conventional mechanisms of exception handling can not be used for many reasons. First, error propagation needs considering the contextual changes that often occur in these systems. Second, error recovery and exception handling strategies also frequently need to be selected according to contextual information. Third, the characterization of an exception may depend on the contextual situation of involved devices. Even though there are now several context-oriented middleware systems that provide support for the development of mobile applications, they rarely provide explicit and adequate features for contextsensitive exception handling. This work presents an analysis of existing exception handling mechanisms, which to some extent consider the context-awareness requirements. Besides, it proposes a general model for context-sensitive exception handling and a supporting mechanism implemented using the MoCA (Mobile Collaboration Architecture) infrastructure. MoCA is a publish-subscribe middleware supporting the development of collaborative mobile applications by incorporating explicit services to empower software agents with contextsensitiveness. Finally, this paper reports our experience in implementing contextaware exception handling strategies in some prototype collaborative applications built with the MoCA system.

\section{Keywords}

Exception handling, mobile computing, context-awareness, middleware, fault tolerance. 


\section{Sumário}

1 Introdução 14

$\begin{array}{ll}\text { 1.1. Problema } & 15\end{array}$

1.2. Limitações dos Trabalhos Relacionados 16

$\begin{array}{ll}\text { 1.3. Solução Proposta } & 17\end{array}$

$\begin{array}{ll}\text { 1.4. Objetivos } & 18\end{array}$

1.5. Organização do Texto 19

2 Sensibilidade ao Contexto 20

2.1. Definições Básicas 21

2.1.1. Contexto e Tipos de Contexto 21

2.1.2. Aplicações Sensíveis ao Contexto 22

2.2. Computação Distribuída Tradicional e Móvel 23

2.3. Middlewares para Aplicações Sensíveis ao Contexto 24

2.3.1. Modelagem de Contexto 25

2.3.2. Paradigmas de Coordenação 26

Baseados em Eventos ou Publish-Subscribe $\quad 27$

Baseados em Espaço de Tuplas $\quad 27$

Reflexão Computacional 28

2.4. Exemplos de Middlewares 29

$\begin{array}{ll}\text { 2.4.1. MoCA } & 29\end{array}$

2.4.2. CAMA 32

2.4.3. CARISMA 34

2.5. Análise dos Middlewares 35

3 Tratamento de Exceções 36

3.1. Aspectos Gerais do Tratamento de Exceções 39

3.1.1. Representação da Exceção 39

3.1.2. Separação entre Exceção Externa e Interna 39

3.1.3. Localização de Tratadores $\quad 40$

3.1.4. Associação de Tratadores 40 
3.1.5. Propagação de Exceção 41

3.2. Aspectos Adicionais Relacionados a Sensibilidade ao Contexto 41

3.2.1. Estudo de caso: Health Care 42

3.2.2. Especificação de Contextos Excepcionais 43

3.2.3. Ausência de Escopos Apropriados para Tratamento de Exceções 44

3.2.4. Necessidade de Tratadores Sensíveis ao Contexto 45

3.2.5. Tratamento de Exceções Imprevistas 45

3.3. Limitações de Alguns Trabalhos Relacionados 46

3.3.1. Tripathi e Miller 46

3.3.2. Souchon, Dony, Urtado e Vauttier 47

3.3.3. lliasov e Romanovsky 48

3.4. Análise das Soluções 49

4 Modelo de Tratamento de Exceções Sensível ao Contexto 51

4.1. Especificação de Contextos Excepcionais 51

4.2. Informação Contextual Extra 52

4.3. Escopos: Dispositivo, Servidor, Regiões ou Grupos 52

4.3.1. Escopos Baseados em Grupos 53

4.3.2. Escopos Baseados em Regiões 53

4.4. Busca Sensível ao Contexto por Tratadores 54

4.5. Tratadores Sensíveis ao Contexto 54

4.6. Propagação Sensível ao Contexto 54

4.7. Tratamento de Exceções Proativo 55

5 Arquitetura de Tratamento de Exceções Sensível ao Contexto 57

5.1. Uma Arquitetura de Software para Tratamento de Exceções 58

5.2. Componentes da Arquitetura $\quad 59$

5.3. Interfaces da Arquitetura 64

5.4. Análise da Arquitetura 69

6 Mecanismo de Tratamento de Exceções Sensível ao Contexto $\quad 70$

6.1. Uso de Aspectos no Mecanismo 70

6.2. Projeto Detalhado dos Componentes 72

6.2.1. Componente ContextualException 72 
6.2.2. Componente HandlingScope 75

6.2.3. Componente Handler 76

6.2.4. Componente ExceptionHandlingStrategy 77

6.2.5. Componente PropagationManager 78

6.2.6. Componente ExceptionPropagation $\quad 79$

6.3. Implementação dos Componentes usando MoCA 81

7 Estudos de Caso $\quad 84$

7.1. Virtual Lines $(\mathrm{VL})$

7.1.1. Especificação de Tratadores e Propagação de Exceções 85

7.1.2. Outras Especificações 88

7.2. Health Care $(\mathrm{HC})$

7.2.1. Exceções Contextuais e Tratadores Sensíveis ao Contexto 91

7.2.2. Propagação Sensível ao Contexto 92

7.2.3. Seqüência de Prioridade Entre os Escopos 94

7.2.4. Tratadores Sensíveis ao Contexto 95

7.2.5. Propagação de Exceções Proativa 96

7.3. Análise do Mecanismo 97

8 Conclusões 100

8.1. Discussões Finais 100

8.2. Contribuições 102

8.3. Trabalhos Futuros 102

9 Referências Bibliográficas 104 


\section{Lista de Figuras}

Figura 1. Arquitetura MoCA 30

Figura 2. Dependências entre as APIs de MoCA 32

Figura 3. Principais Abstrações de CAMA 32

Figura 4. Arquitetura CARISMA 35

Figura 5. Componente Tolerante a Falhas Ideal 37

Figura 6. Arquitetura do Mecanismo de Tratamento de Exceções 62

Figura 7. Componente ContextualException e Interfaces 64

Figura 8. Componente HandlingScope e Interfaces 65

Figura 9. Componente Handler e Interfaces 66

Figura 10. Componente ExceptionHandlingStrategy e Interfaces $\quad 66$

Figura 11. Componente PropagationManager e Interfaces 67

Figura 12. Componente ExceptionPropagation e Interfaces 68

Figura 13. Projeto do Componente ContextualException 73

Figura 14. Projeto do Componente HandlingScope 75

Figura 15. Projeto do Componente Handler 76

Figura 16. Projeto do Componente ExceptionHandlingStrategy $\quad 77$

Figura 17. Projeto do Componente PropagationManager 79

Figura 8. Projeto do Componente ExceptionPropagation 80

Figura 19. Adição de Contextos Excepcionais 81

Figura 20. Observador de Eventos para Detecção de Contextos $\quad 82$

Figura 21. Recuperação dos Dispositivos do Escopo de Região 82

Figura 22. Propagação de Exceções 83

Figura 23. Implementação de ConfigurationServer em VL 88

Figura 24. Implementação de ConfigurationClient em VL 89

Figura 25. Implementação de NotifyHandler em VL 90

Figura 26. Implementação de ConfigurationClient em HC 93

Figura 27. Implementação de NotifyHandler em HC 94

Figura 28. Estratégia para Busca de Tratadores de HeartAttack em HC 95

Figura 29. Implementação de FirstHelp em HC 96

Figura 30. Definição de Propagação Proativa em HC 97 


\section{Lista de Tabelas}

Tabela 1. Componentes e suas Responsabilidades na Arquitetura $\quad 60$

Tabela 2. Tratadores e Escopos Associados em VL 87

Tabela 3. Exceção Contextual HeartAttack e seus Tratadores em HC 91 


\section{Lista de Siglas e Abreviaturas}

CAMA Context-Aware Mobile Agents

CARISMA Context-Aware Reflective mlddleware System for Mobile Applications

HC Health Care

MoCA Mobile Collaboration Architecture

VL Virtual Lines 